\title{
Plasmonic Photothermal Fluorescence Modulation for Homogeneous Biosensing
}

\author{
Jesica V. Pellegrotti, ${ }^{\dagger}$ Emiliano Cortés, ${ }^{\dagger}$ Martin D. Bordenave, ${ }^{\dagger}$ Martin Caldarola, ${ }^{\dagger}$ Mark P. Kreuzer, ${ }^{\dagger}$
} Alfredo D. Sanchez, ${ }^{\dagger}$ Ignacio Ojea, ${ }^{\S}$ Andrea V. Bragas, ${ }^{\dagger}$ and Fernando D. Stefani ${ }^{*},{ }^{\dagger}, \pm 0$

${ }^{\dagger}$ Centro de Investigaciones en Bionanociencas (CIBION), Consejo Nacional de Investigaciones Científicas y Técnicas (CONICET), Godoy Cruz 2390, C1425FQD Buenos Aires, Argentina

"Departamento de Física and ${ }^{\S}$ Departamento de Matemática \& Instituto de Investigaciones Matemáticas "Luis A. Santaló" (IMAS, CONICET), Facultad de Ciencias Exactas y Naturales, Universidad de Buenos Aires, Pabellón 1 Ciudad Universitaria, C1428EHA Buenos Aires, Argentina

\section{Supporting Information}

ABSTRACT: Fluorescence readout is uniquely powerful for biological assays and imaging because it combines the detection of specific biotargets with high spatial and temporal resolution. Recently, several strategies for the modulation in time of fluorescence emission have been proven useful to separate the target signal from constant background contributions. Here, we investigate the emission modulation of organic fluorophores located in the nanometric vicinity of plasmonically heated gold nanorods and apply it to a novel, all-optical homogeneous biosensing scheme. The combination of plasmonic heating and temperature sensitive molecular fluorescence enables the robust quantification of surface reactions.

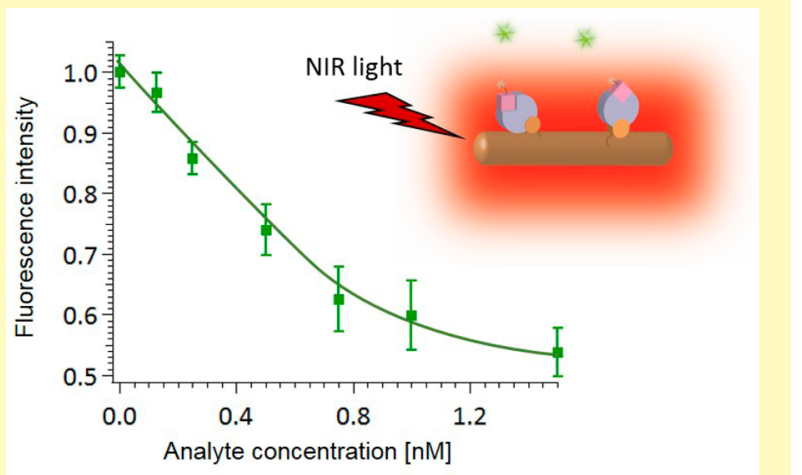

KEYWORDS: plasmonic heating, gold nanorods, gold nanoparticle, fluorescence quenching, sandwich assay

$\mathrm{F}$ luorescence labeling and detection provide high sensitivity and temporal resolution for biological imaging and clinical assays. In low background conditions, single fluorescent molecules are easily detected and tracked. Biological samples usually contain endogenous fluorophores which generate a background contribution that limits the sensitivity for detection of the fluorescence of interest. One way to overcome this obstacle is making brighter and more stable fluorophores either by $\operatorname{design}^{1-3}$ or through control of their chemical ${ }^{4,5}$ or physical $^{6,7}$ environment. Another way is using markers with long-lived excited states whose emission can be discerned from the background in time-resolved measurements. ${ }^{8,9}$ Alternatively, various strategies have been investigated to specifically modulate the fluorescence emission of molecules. A controlled modulation enables the distinction of the target fluorescence signal from continuous background. ${ }^{10}$ For example, acoustooptic modulation of fluorescence has been applied to imaging in turbid media. ${ }^{11,12}$ Optically modulated fluorescence signals were obtained from organic fluorophores and fluorescent proteins making use of photoisomerization reactions ${ }^{13,14}$ and from DNA-encapsulated Ag clusters via the excitation of dark states with near-infrared light. ${ }^{15}$ The emission of FRET pairs can be modulated optically by depleting the ground state of the acceptor $^{16}$ or quenching its emission. ${ }^{19}$

Here, we propose another way of modulating optically the fluorescence emission of molecules near metallic nanoparticles.
Metallic nanoparticles are extremely efficient light-to-heat converters when they are illuminated with wavelengths at their surface plasmon resonances. ${ }^{18-20}$ Plasmonic heating enables the control of high temperature fields around nanoparticles, ${ }^{21-24}$ and has found numerous applications such as controlled release of molecules, ${ }^{25}$ DNA melting assays, ${ }^{26}$ nanoscale thermodynamics ${ }^{21}$ and catalysis, ${ }^{27}$ nanoparticle detection, $^{28}$ and photothermal therapy. ${ }^{29}$ Additionally, the fluorescence emission of most organic fluorophores is affected by temperature. Higher temperatures usually lead to lower quantum yields because of the thermal activation of nonradiative decay channels. ${ }^{30}$ The quantum efficiency of most organic fluorophores decreases with temperature at rates as high as $1-2 \% / \mathrm{K}^{31,32}$ This effect has been long known ${ }^{32,33}$ and applied to diverse optical methods of temperature determination. ${ }^{34-38} \mathrm{We}$ investigate the combination of these phenomena to modulate the fluorescence emission of organic fluorophores placed in the nanometric vicinity of gold nanorods (AuNRs) and demonstrate its application for homogeneous biosensing.

A sketch depicting the concept of plasmonic photothermal modulation of fluorescence is shown in Figure 1a. The spectra

Received: August 18, 2016

Accepted: November 7, 2016

Published: November 7, 2016 


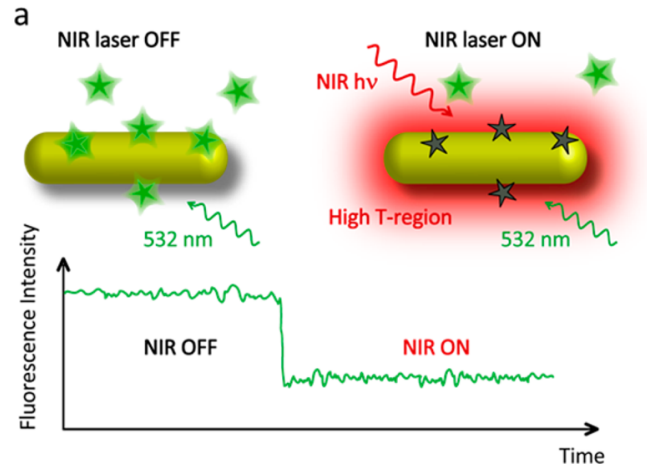

$\mathrm{b}$

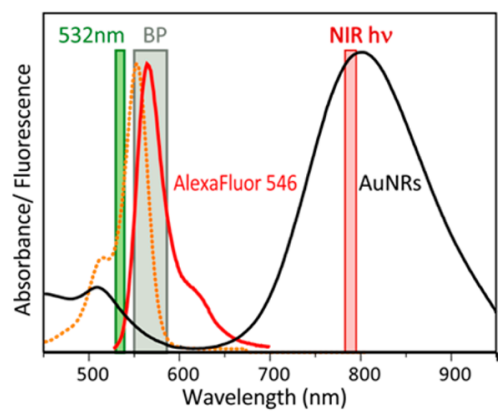

Figure 1. Scheme of the photothermal fluorescence quenching concept. (a) Upon illumination with near-infrared (NIR) light tuned to the surface plasmon resonance of the AuNRs, the fluorescence emission of fluorophores bound to the AuNRs is quenched due to the local high temperature. (b) Absorption (orange) and emission (red) spectra of AlexaFluor546. Extinction spectra (black) of the AuNRs (size $90 \mathrm{~nm} \times 22 \mathrm{~nm}$ ) in water showing the longitudinal plasmon mode at $800 \mathrm{~nm}$ and the transverse one at $525 \mathrm{~nm}$. The vertical bands indicate the spectral regions used for fluorescence excitation (laser at $532 \mathrm{~nm}$ ), fluorescence detection (BP filter), and plasmonic heating (laser at $785 \mathrm{~nm}$ ).
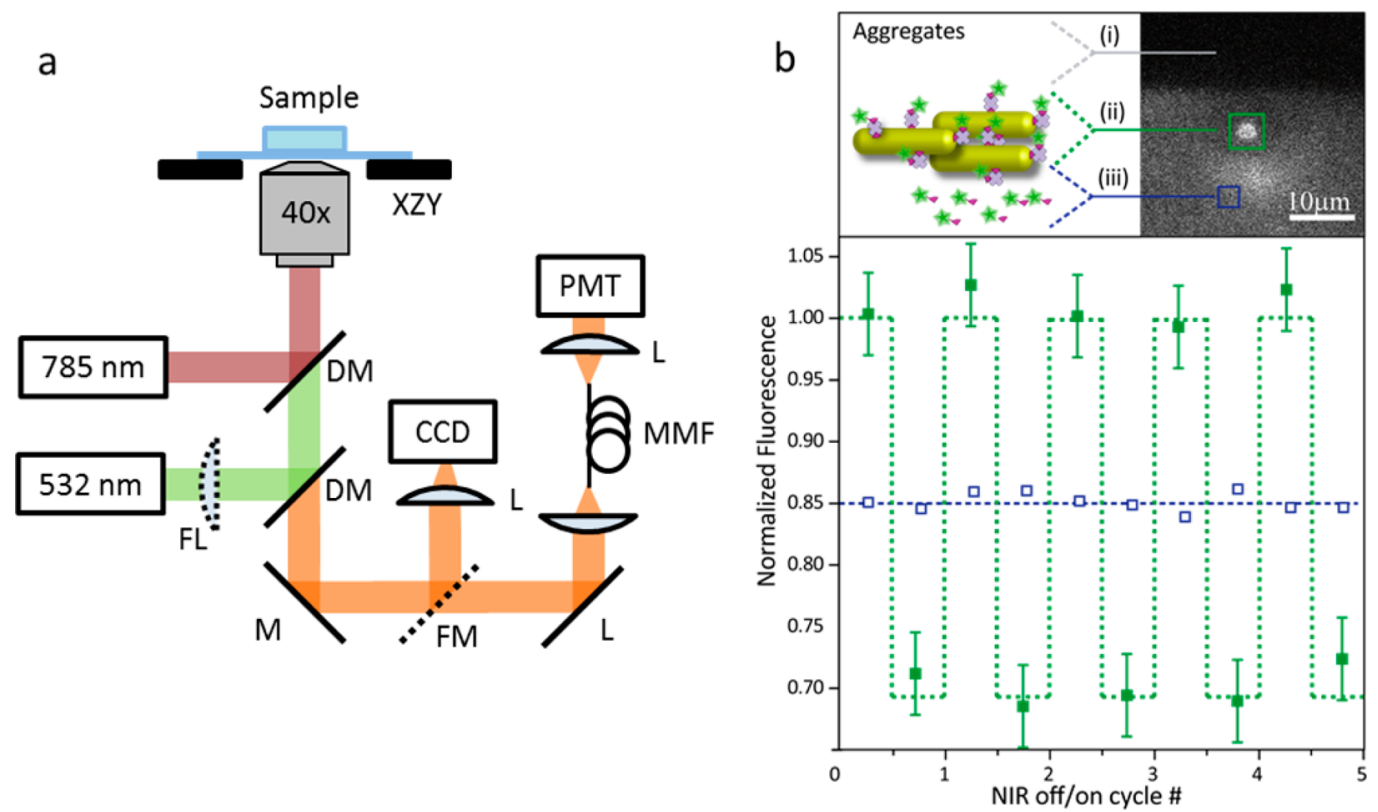

Figure 2. (a) Scheme of the experimental setup. DM: dichroic mirror, M: mirror, L: lens, MMF: multimode fiber, PMT: photomultiplier tube, CCD: charge coupled device. The flippable mirror and lens (FM and FL) permit switching between confocal and wide-field operations. (b) Schematic and epifluorescence image of the samples showing regions of (i) dark background, (ii) an aggregate of fluorescently labeled AuNRs, and (iii) free fluorophores. Fluorescence intensity detected on regions (ii) (green) and (iii) (blue) during several off/on cycles of the NIR heating laser.

of the fluorophore and AuNRs used are shown in Figure $1 \mathrm{~b}$. The sample contains fluorescent species both free in solution and bound to the surface of the AuNRs. Upon illumination with light of a wavelength tuned to the longitudinal surface plasmon resonance of the AuNRs, the fluorophores bound to the AuNRs emit less fluorescence due to the local increase in temperature.

We note that fluorescence emission may be suppressed simply by the presence of the AuNRs in close proximity, due to direct energy transfer from the excited molecules to the metal. ${ }^{18}$ In this study all measurements take as baseline the fluorescence emission of fluorophores already bound to the AuNRs. In this way we investigate exclusively the reduction of fluorescence induced by the plasmonic heating.

Switching on and off the light used for plasmonic heating, it is possible to modulate specifically the fluorescence emission of molecules bound to the AuNRs, and thereby tell it apart from the constant fluorescence background. In order to achieve the highest modulation amplitude, the light used for plasmonic heating should not contribute to fluorescence excitation, as this would counteract the photothermal quenching. To fulfill this condition, we used AuNRs with an aspect ratio of 1:4 and a longitudinal surface plasmon resonance at around $800 \mathrm{~nm}$ (Figure 1b). ${ }^{39}$ In this way, plasmonic heating was performed with near-infrared light, leaving the visible region of the spectrum available for fluorescence excitation and detection. As a suitable fluorescent probe we used AlexaFluor546 (Figure 1b). We investigated the performance of AuNRs with aspect ratio 1:4 and two average sizes: $40 \mathrm{~nm} \times 10 \mathrm{~nm}$ and $90 \mathrm{~nm} \times$ $22 \mathrm{~nm}$ (see Figure S1 for electron microscopy images).

Plasmonic Photothermal Fluorescence Quenching. The first experiment was designed to verify whether the proposed mechanism for fluorescence modulation was in fact feasible. The basic components of the experimental setup are shown in Figure 2a. It consisted of a custom-made fluorescence microscope that can be operated in wide field as well as in 
confocal mode, with an additional laser used for plasmonic heating of the AuNRs. A sample was prepared where three regions could be identified (Figure $2 \mathrm{~b}$ ): (i) dark background with no fluorophores, (ii) aggregates of $90 \mathrm{~nm} \times 22 \mathrm{~nm}$ AuNRs labeled with AlexaFluor546, and (iii) free AlexaFluor546 fluorophores (see the Materials and Methods section for details). While the fluorescence emission was interrogated constantly, we intermittently illuminated the different regions of the sample with the NIR laser. The NIR laser produced a clear modulation in the emission intensity of the fluorescently labeled AuNRs, whereas on the free dyes it made no observable change (Figure $2 \mathrm{~b}$ ). The photothermal modulation of the fluorescence is directly visible as shown in Video 1. Remarkably, no degradation of the fluorescence intensity was observed after several heating cycles with $400 \mathrm{~kW} / \mathrm{cm}^{2}$ irradiance.

This experiment demonstrates that it is indeed possible to modulate optically the fluorescence emission of fluorophores bound to AuNRs by means of plasmonic heating. We note however that in this experiment, the AuNRs were deposited in clusters of unknown numbers of particles and interparticle distance which hinders the comparison to simulations. In order to gain further insight, we performed simulations and experiments on individual fluorescently labeled AuNRs.

Simulations and Measurements on Single AuNRs. In the absence of phase transformations, the temperature increase in the vicinity of a nanoparticle can be described by the heat transfer equation:

$$
\rho(\mathbf{r}) c(\mathbf{r}) \frac{\partial T(\mathbf{r}, t)}{\partial t}=\nabla k(\mathbf{r}) \nabla T(\mathbf{r}, t)+Q(\mathbf{r}, t)
$$

where $\mathbf{r}$ and $t$ are the spatial and time coordinates, $T(\boldsymbol{r}, t)$ is the local temperature. All experimental parameters are well-known. $\rho(\boldsymbol{r}), c(\boldsymbol{r})$, and $k(\boldsymbol{r})$ are the mass density, specific heat, and thermal conductivity of the surrounding water, respectively. $Q(r, t)$ is the power energy absorbed by the AuNR, which can be expressed as

$$
Q=\sigma_{\mathrm{abs}} I
$$

where $\sigma_{\mathrm{abs}}$ is the absorption cross section, and $I$ the irradiance of the incident light. We solved eq 1 numerically in order to obtain the time-resolved temperature field around AuNRs. As an example, we show in Figure $3 a$ the calculated steady-state temperature field around an AuNR of $90 \mathrm{~nm} \times 22 \mathrm{~nm}$ immersed in water and illuminated with $600 \mathrm{~kW} / \mathrm{cm}^{2}$ of CW radiation at $785 \mathrm{~nm}$. The temperature of water around the AuNR is increased in a nanometric region around the nanoparticle, and decreases to the bulk value within a few tens of nanometers in all directions. The calculated temperature field around a smaller AuNR of $40 \mathrm{~nm} \times 10 \mathrm{~nm}$ under identical illumination in shown in Figure S2. Absorption cross sections at $785 \mathrm{~nm}$ of $\sigma_{40 \mathrm{~nm} \times 10 \mathrm{~nm}}=3.8 \times 10^{-4} \mu \mathrm{m}^{2}$ and $\sigma_{90 \mathrm{~nm} \times 22 \mathrm{~nm}}=2.4 \times$ $10^{-3} \mu \mathrm{m}^{2}$, for the smaller and larger AuNRs were used, respectively, according to information provided by the manufacturer of the AuNRs and FDFD numerical simulations.

Values of the temperature near the surface of the larger AuNRs for a range of irradiances are shown in Figure $3 \mathrm{~b}$. The time evolution of the temperature at two positions near the AuNR is shown in Figure 3c; the steady state is reached in about $100 \mathrm{~ns}$, which is practically instantaneous for most applications. The smaller AuNRs show an analogous plasmonic heating behavior.

The temperature response of the fluorophore AlexaFluor546 was characterized by measuring its fluorescence emission at
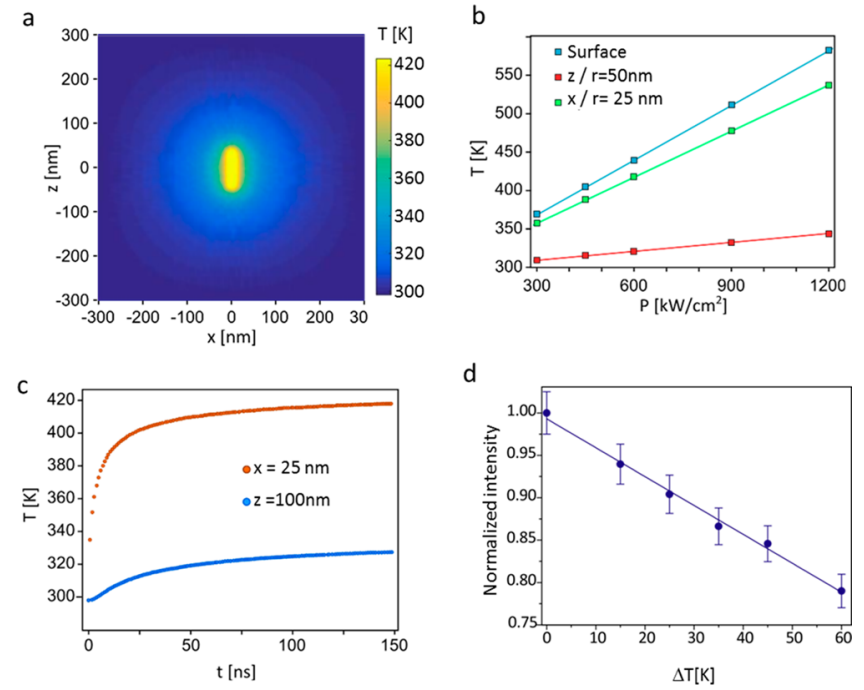

Figure 3. (a) Temperature field around a AuNR of $90 \mathrm{~nm} \times 22 \mathrm{~nm}$ illuminated with $600 \mathrm{~kW} / \mathrm{cm}^{2}$ at $785 \mathrm{~nm}$. (b) Temperature as a function of the irradiance at different $(x, z)$ positions near the AuNR: at its surface, at $3 \mathrm{~nm}$ from its surface in the $x$ direction, and at $5 \mathrm{~nm}$ from its surface in the $z$ direction. (c) Time evolution of the temperature at two different positions near the AuNR. (d) Temperature response of AlexaFluor546 in solution.

different temperatures in a spectrofluorometer. Figure $3 \mathrm{~d}$ shows the fluorescence emission, integrated in the band $550-580 \mathrm{~nm}$, as a function of temperature. We observed a linear decrease of $0.35 \% / \mathrm{K}$, in agreement with previous reports for other Rhodamine derivative fluorophores. ${ }^{37,40}$ Combining the temperature response of the fluorophore with the calculated temperature fields, it is possible to estimate the decrease in fluorescence emission of fluorophores bound to the AuNRs when the latter is optically heated. This modeled signal reduction can then be compared to measurements performed on single fluorescently labeled AuNRs (Figure 4).

We characterized the photothermal fluorescence reduction of individual AuNRs of $90 \mathrm{~nm} \times 22 \mathrm{~nm}$ and $40 \mathrm{~nm} \times 10 \mathrm{~nm}$, both labeled with AlexaFluor546. The optical heating was induced with $400 \mathrm{~kW} / \mathrm{cm}^{2}$ irradiance of a NIR laser modulated at $1 \mathrm{~Hz}$. Figure $4 \mathrm{~b}$ shows the mean fluorescence intensity of labeled AuNRs measured over 10 different individual NPs of each size. As the NIR laser is switched on and off, reproducible oscillations were observed for the individual AuNRs, with amplitudes varying less than 5\% from AuNR to AuNR. As expected from their absorption cross sections $\left(\sigma_{40 \mathrm{~nm} \times 10 \mathrm{~nm}}=3.8\right.$ $\times 10^{-4} \mu \mathrm{m}^{2}$ and $\left.\sigma_{90 \mathrm{~nm} \times 22 \mathrm{~nm}}=2.4 \times 10^{-3} \mu \mathrm{m}^{2}\right)$, the modulation amplitude is greater for the larger AuNRs. The actual value of the modulation observed for each size of AuNRs agrees well with the prediction based on the calculated temperature field and the bulk temperature response of the fluorophore (Figure $4 a, b)$. We note that the photothermal modulation of fluorescence found in the AuNR clusters was considerably larger (Figure 2b). This can be explained by taking into account collective effects due to particles agglomeration. ${ }^{41}$

Application to Homogeneous Biosensing. Homogenous biosensing assays consist of mixing the reagents with the sample in one phase, typically liquid, incubating them for a certain time according to protocol, and finally making the measurement. They are simple to implement, but usually their sensitivity is limited by background contributions. Heterogeneous assays where the analyte is selectively transferred to a 

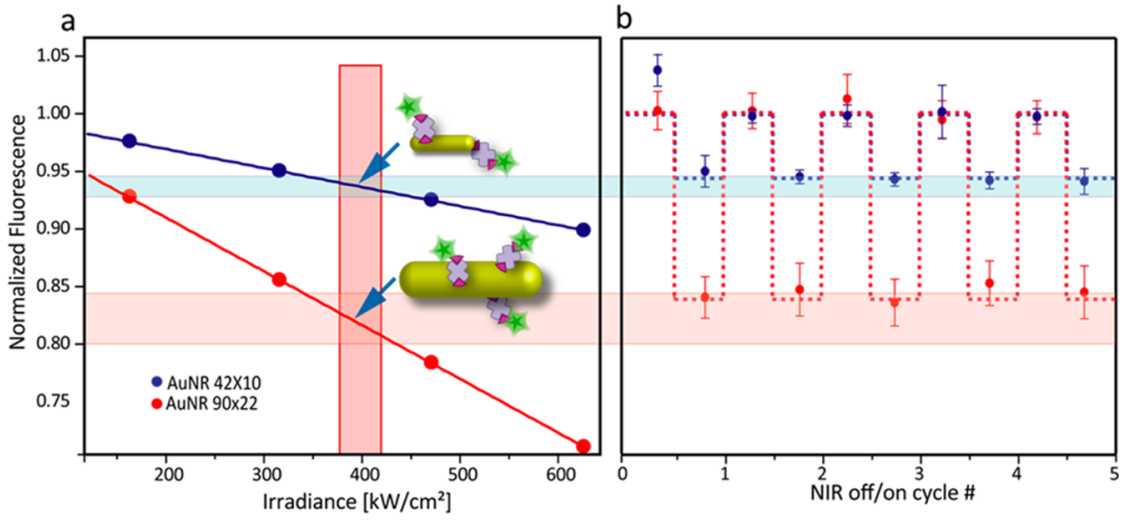

Figure 4. (a) Simulation of the reduction in fluorescence intensity expected for AlexaFluor546 bound to AuNRs $(40 \mathrm{~nm} \times 10 \mathrm{~nm}$ in blue, $90 \mathrm{~nm} \times$ $22 \mathrm{~nm}$ in red) as a function of the irradiance at $785 \mathrm{~nm}$. (b) Average fluorescence intensity of individual AuNRs $(40 \mathrm{~nm} \times 10 \mathrm{~nm}$ in blue, $90 \times 22$ $\mathrm{nm}^{2}$ in red) conjugated to AlexaFluor546 over several cycles of on/off switching of the heating laser. A comparison to the simulation is made over a range of irradiances accounting for the size distribution of the AuNRs and separation distances fluorophore-AuNR ranging from 10 to $14 \mathrm{~nm}$.

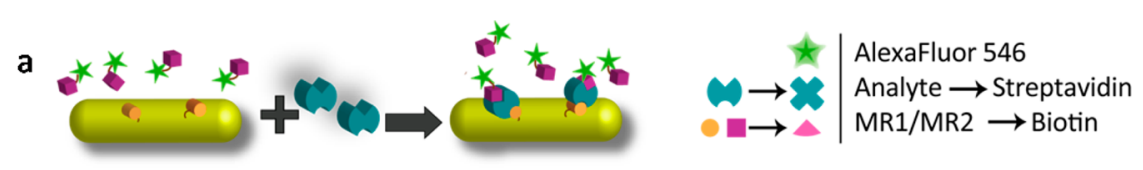

b

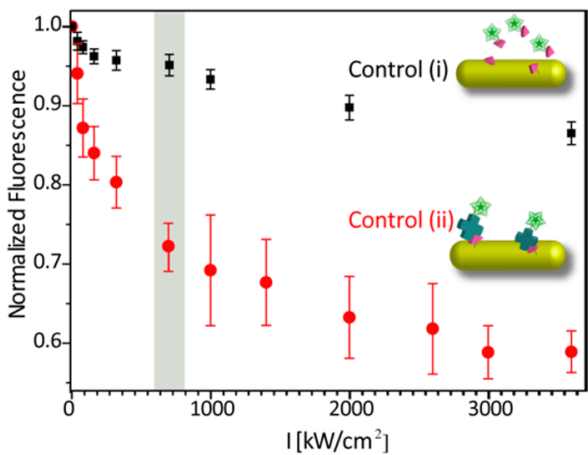

C

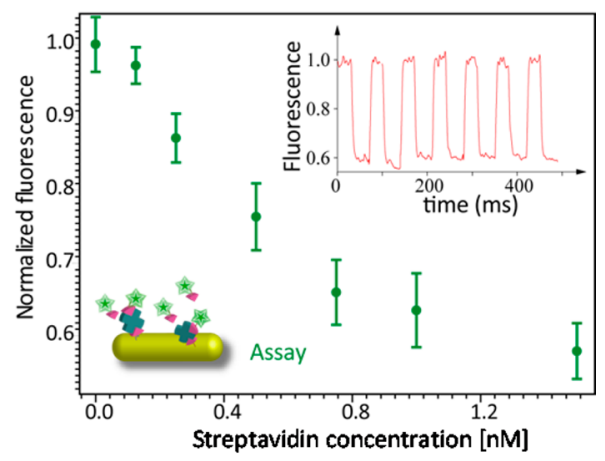

Figure 5. Sandwich assay based on photothermal quenching. (a) Representation of the proposed biosensor and the components used in our experimental demonstration. (b) Effect of NIR illumination on the fluorescence emission of AlexaFluor546 free in solution or unspecifically bound to the AuNRs (control (i), black) and specifically attached to AuNRs (control (ii), red). (c) Normalized fluorescence emission under NIR illumination as a function of streptavidin concentration obtained for assays composed of $1 \mathrm{nM}$ biotin-AuNRs $(90 \mathrm{~nm} \times 22 \mathrm{~nm})+5 \mathrm{nM}$ biotinAlexaFluor546. Inset: time trace of fluorescence signal over multiple on-off cycles of the NIR laser for the case of [Streptavidin] $=0.75 \mathrm{nM}$.

different phase, typically a substrate, offer a way out to remove background contributions from the remaining reagents and other sample components. In this way, heterogeneous assays achieve higher sensitivities at the expense of more complex methodologies, involving fluid exchange and/or physical separation steps.

The photothermal modulation of fluorescence is a phenomenon specific to fluorophores bound to the AuNRs that can be addressed in bulk suspension without any need of separating the nanoparticles from the liquid. Therefore, it is useful for application in simple homogeneous assays to discern (and quantify) the target signal, from constant background contributions from fluorophores free in solution and any other component of the sample.

Figure 5a shows schematically the components and working principle of such an assay. AuNRs are functionalized with a molecular recognition unit (MR1) specific for the target analyte. A different (or the same) molecular recognition unit (MR2) is conjugated to a suitable fluorophore. In the presence of the analyte, the molecular sandwich MR1-analyte-MR2 forms, bringing the fluorophores to the vicinity of the AuNRs where their fluorescence emission can be reduced by plasmonic heating (Figure 5a). By quantifying the reduction of fluorescence induced by a certain irradiance of the NIR laser, it is possible to determine the concentration of analyte by comparison to a calibration curve.

For an experimental test, we used the biotin-streptavidin recognition pair. AuNRs $(90 \mathrm{~nm} \times 22 \mathrm{~nm})$ and AlexaFluor546 were functionalized with biotin, in order to detect the presence of streptavidin (Figure 5a). For these measurements in solution, the setup was used in confocal mode (Figure 1a), with the observation volume restricted to about $1 \mathrm{fL}$.

In order to find the optimum level of irradiance of the NIR heating laser the detection of the specific signal, we performed measurements on two control samples: (i) biotinylated AuNRs and AlexaFluor546 in the absence of streptavidin and (ii) biotinylated AuNRs previously incubated with excess of AlexaFluor546-streptavidin. On each sample we monitored the reduction in fluorescence emission as a function of the irradiance with the NIR laser (Figure 5b). On the control (i) 
we observed a small nonspecific reduction of fluorescence that reaches at value of $10 \%$ for $3500 \mathrm{~kW} / \mathrm{cm}^{2}$. This reduction of fluorescence intensity induced by the NIR laser is attributed to nonspecific binding and/or stimulated emission depletion. Bulk heating of the solution can be neglected based on calculations and the fact that the solution temperature remained unchanged during the course of the experiment. On sample (ii), the reduction of fluorescence induced by the NIR laser is considerably larger at all levels of irradiance, and we identified two regimes. Up to approximately $650 \mathrm{~kW} / \mathrm{cm}^{2}$ the fluorescence emission is reduced strongly as a function of the irradiance, and with magnitudes consistent with calculations. For higher levels of irradiance the fluorescence reduces at a lower rate and the variability between measurements increases, probably because parallel effects might be taking place, such as local boiling of the solvent and formation of microbubbles. ${ }^{42}$

Based on the comparison of the dependencies on irradiance of the specific and the nonspecific signals, we performed a biorecognition assay at $600 \mathrm{~kW} / \mathrm{cm}^{2}$, as follows. We used biotin-AuNRs and biotin-AlexaFluor546 to quantify the concentration of unlabeled streptavidin in solution. In Figure $5 \mathrm{c}$, a calibration curve is shown using $1 \mathrm{nM}$ AuNRs, $5 \mathrm{nM}$ biotin-AlexaFluor 546, and additions of streptavidin in known concentrations. Each data point corresponds to the average of 5 replications of the assays; in turn, each assay consisted of measuring the average fluorescence reduction over 10 on-off cycles of the NIR laser performed in less than $1 \mathrm{~s}$ (Figure 5c inset). Under these conditions, the assay presents a limit of detection of about $250 \mathrm{pM}$, limited partially by variance due to pipetting errors from assay to assay.

These experiments based on the biotin-streptavidin model demonstrate that the mechanism of fluorescence modulation by plasmonic heating enables robust homogeneous biosensing. They also make evident some improvements for future implementations and show that the sensitivity could be improved considerably. The assay presented a linear response up to $800 \mathrm{pM}$. Such an early saturation at a concentration of streptavidin, practically identical to the concentration of AuNRs, indicates that the AuNRs had too few, on the order of one active biotin site on their surface, which is far from the actual capacity of the AuNRs. An optimized surface functionalization should lead to working ranges 10 - to 100 fold greater. The sensitivity of $250 \mathrm{pM}$ could be improved considerably if the nonspecific signal is reduced through, for example, (i) a surface functionalization of AuNRs that minimizes nonspecific binding, and (ii) the use fluorophores unable to undergo stimulated emission depletion of their excited state at the wavelength used for plasmonic heating. Another possible future improvement to achieve higher sensitivity is the application of lock-in detection synchronized to the modulation of the NIR.

\section{CONCLUSIONS}

In summary, we have demonstrated that the local increase of temperature produced by plasmonic heating of AuNRs can selectively quench the fluorescence emission of fluorophores at their surface. This combination of plasmonic heating and temperature sensitive molecular fluorescence enables the robust quantification of surface reactions in all-optical homogeneous sandwich assays.

Based on the rich library of protocols for the surface functionalization of gold nanoparticles and for the fluorescent labeling of proteins and antibodies, this novel sensing mechanism is applicable to numerous analytes. Also, the approach is not limited to gold nanorods. With a suitable fluorophore, it is possible to use nanoparticles of various shapes and compositions. Multiplexing can be envisaged by different fluorescence readout or different heating wavelengths.

Finally, we remark that the photothermal quenching mechanism is independent of electromagnetic quenching that may occur upon binding of the fluorescent biorecognition unit to the AuNRs, which has already been applied in other assays. ${ }^{43}$ Therefore, an assay combining both effects should in principle lead to a higher sensitivity.

\section{MATERIALS AND METHODS}

Biotin-functionalized gold nanorods (AuNRs) with an aspect ratio of about 4.3 and a longitudinal plasmon resonance centered at $808 \mathrm{~nm}$ were purchased from Nanopartz, inc. Phosphate buffered saline (PBS) and AlexaFluor546 conjugated to biocitin and streptavidin were purchased from Invitrogen.

Sample Preparation for Demonstration of Selective Plasmonic Photothermal Fluorescence Quenching. Solutions containing $20 \mathrm{nM}$ AlexaFluor546-X (X: biocitin or streptavidin) and 1 $\mathrm{nM}$ AuNRs were prepared in PBS with $0.1 \%$ bovine serum albumin (BSA). Solutions were left to react for $2 \mathrm{~h}$ and then an aliquot was taken to be measured.

Sample Preparation for the Streptavidin Sandwich Assay. Suspensions of $1 \mathrm{nM}$ AuNRs $(90 \mathrm{~nm} \times 22 \mathrm{~nm})$ were incubated overnight at $4{ }^{\circ} \mathrm{C}$ with different concentration of streptavidin and $0.1 \%$ BSA. AlexaFluor546-biotin was added to final concentrations of $5 \mathrm{nM}$ or $80 \mathrm{nM}$, depending on the experiment. The final solution was left to react for $2 \mathrm{~h}$ and then an aliquot was taken to be measured.

Fluorescence and UV-vis Absorption Measurements. These were performed in a fluorescence spectrophotometer (Cary Eclipse Fluorimeter) and in a UV-vis Spectophotometer (Cary Varian 50 UV-vis, Agilent), respectively.

Photothermal Fluorescence Quenching Measurements. These were carried out in an inverted fluorescence confocal microscope mode with an additional laser beam used for plasmonic heating. All measurements were carried out in solution using a microwell slide (Hamamatsu) as sample chamber of $25 \mu \mathrm{L}$. The chamber was covered with another cover glass during the measurements in order to prevent from evaporation. For fluorescence excitation a frequency doubled Nd:YAG laser at $532 \mathrm{~nm}$ was used (Coherent Compass $315 \mathrm{M}$ ). For plasmonic heating of the AuNRs a CW Ti-Sa laser (KMLabs) tuned at $785 \mathrm{~nm}$ was used. NIR illumination was switched on and off with a mechanical shutter. The used microscope objective was a 40× NA 0.9 (Olympus ULPSAPO). For confocal detection a photomultiplier tube (Hamamatsu R928) was used, and for wide-field a CCD device (Andor iXon 897).

Calculations of the Temperature around the Gold Nanorod. These were made solving numerically in three dimensions the heat equation $\mathrm{d} u / \mathrm{d} t=\Delta u+f$ in a cubic box of $300 \times 300 \times 300 \mathrm{~nm}$. On the boundary of the box, a constant temperature equal to room temperature $(298 \mathrm{~K})$ was imposed. The nanorod was centered at the origin. The source $f$ was modeled as a delta function, taking a positive value determined by the absorption cross section of the gold nanorod (as provided by the manufacturer) and the heating laser power density, and placed at the center of the nanorod, and being zero elsewhere. The heat equation was solved using the Finite Element Method in space along with a finite difference discretization on time, for a total time of $150 \mathrm{~ns}$. A $1 \mathrm{~ns}$ time step was taken. A tetrahedral mesh was generated manually. The mesh is refined inside the nanorod and in its close vicinity, in order to minimize the error produced by the jump of the diffusive coefficient at the interface between gold and water. The distance between nodes of the mesh near the rod is about $2 \mathrm{~nm}$, and it grows linearly with the distance to the rod. 


\section{ASSOCIATED CONTENT}

\section{S Supporting Information}

The Supporting Information is available free of charge on the ACS Publications website at DOI: 10.1021/acssensors.6b00512.

Electron microscopy images of the AuNRs and calculated temperature field distributions (PDF)

Plasmonic photothermal modulation of fluorescence emission from a cluster of gold nanorods labeled with AlexaFluor546 (AVI)

\section{AUTHOR INFORMATION}

\section{Corresponding Author}

*E-mail: fernando.stefani@cibion.conicet.gov.ar. ORCID

Fernando D. Stefani: 0000-0002-3277-7215

Notes

The authors declare no competing financial interest.

\section{ACKNOWLEDGMENTS}

This project was funded with the support of the following grants: CONICET PIO 13320130100199CO, ANCYPT PICT2009-0110, PICT-2010-2511, PICT-2013-0792, and a Partner Group of the Max-Planck-Society. We thank Lia Pietrasanta, Julian Gargiulo, and Federico Barabas for experimental assistance. CONICET, FDS, and JVP have filed a provisional patent application on the described method for molecular sensing.

\section{REFERENCES}

(1) Arden-Jacob, J.; Frantzeskos, J.; Kemnitzer, N. U.; Zilles, A.; Drexhage, K. H. New Fluorescent Markers for the Red Region. Spectrochim. Acta, Part A 2001, 57 (11), 2271-2283.

(2) Avlasevich, Y.; Müller, S.; Erk, P.; Müllen, K. Novel CoreExpanded Rylenebis(dicarboximide) Dyes Bearing Pentacene Units: Facile Synthesis and Photophysical Properties. Chem. - Eur. J. 2007, 13 (23), 6555-6561.

(3) Altman, R. B.; Terry, D. S.; Zhou, Z.; Zheng, Q.; Geggier, P.; Kolster, R. a; Zhao, Y.; Javitch, J. a; Warren, J. D.; Blanchard, S. C. Cyanine Fluorophore Derivatives with Enhanced Photostability. Nat. Methods 2011, 9 (1), 68-71.

(4) Wu, X. M.; Zhu, W. H. Stability Enhancement of Fluorophores for Lighting up Practical Application in Bioimaging. Chem. Soc. Rev. 2015, 44 (13), 4179-4184.

(5) Vogelsang, J.; Kasper, R.; Steinhauer, C.; Person, B.; Heilemann, M.; Sauer, M.; Tinnefeld, P. A Reducing and Oxidizing System Minimizes Photobleaching and Blinking of Fluorescent Dyes. Angew. Chem., Int. Ed. 2008, 47 (29), 5465-5469.

(6) Pellegrotti, J. V.; Acuna, G. P.; Puchkova, A.; Holzmeister, P.; Gietl, A.; Lalkens, B.; Stefani, F. D.; Tinnefeld, P. Controlled Reduction of Photobleaching in DNA Origami-Gold Nanoparticle Hybrids. Nano Lett. 2014, 14 (5), 2831-2836.

(7) Puchkova, A.; Vietz, C.; Pibiri, E.; Wünsch, B.; Sanz Paz, M.; Acuna, G. P.; Tinnefeld, P. DNA Origami Nanoantennas with over 5000-Fold Fluorescence Enhancement and Single-Molecule Detection at $25 \mathrm{Mm}$. Nano Lett. 2015, 15 (12), 8354-8359.

(8) Marriott, G.; Clegg, R. M.; Arndt-Jovin, D. J.; Jovin, T. M. Time Resolved Imaging Microscopy. Phosphorescence and Delayed Fluorescence Imaging. Biophys. J. 1991, 60 (6), 1374-1387.

(9) Dahan, M.; Laurence, T.; Pinaud, F.; Chemla, D. S.; Alivisatos, a P.; Sauer, M.; Weiss, S. Time-Gated Biological Imaging by Use of Colloidal Quantum Dots. Opt. Lett. 2001, 26 (11), 825-827.
(10) Hsiang, J.-C.; Jablonski, A. E.; Dickson, R. M. Optically Modulated Fluorescence Bioimaging: Visualizing Obscured Fluorophores in High Background. Acc. Chem. Res. 2014, 47 (5), 1545-1554.

(11) Kobayashi, M.; Mizumoto, T.; Shibuya, Y.; Enomoto, M.; Takeda, M. Fluorescence Tomography in Turbid Media Based on Acousto-Optic Modulation Imaging. Appl. Phys. Lett. 2006, 89 (18), 181102.

(12) Zhang, Y.; Zhang, K.; Wang, J.; Tian, Z.; Li, A. D. Photoswitchable Fluorescent Nanoparticles and Their Emerging Applications. Nanoscale 2015, 7 (46), 19342-19357.

(13) Mahoney, D. P.; Owens, E. A.; Fan, C.; Hsiang, J. C.; Henary, M. M.; Dickson, R. M. Tailoring Cyanine Dark States for Improved Optically Modulated Fluorescence Recovery. J. Phys. Chem. B 2015, 119 (13), 4637-4643.

(14) Marriott, G.; Mao, S.; Sakata, T.; Ran, J.; Jackson, D. K.; Petchprayoon, C.; Gomez, T. J.; Warp, E.; Tulyathan, O.; Aaron, H. L.; et al. Optical Lock-in Detection Imaging Microscopy for ContrastEnhanced Imaging in Living Cells. Proc. Natl. Acad. Sci. U. S. A. 2008, 105 (46), 17789-17794.

(15) Hsiang, J.-C.; Fleischer, B. C.; Dickson, R. M. Dark StateModulated Fluorescence Correlation Spectroscopy for Quantitative Signal Recovery. J. Phys. Chem. Lett. 2016, 7, 2496-2501.

(16) Richards, C. I.; Hsiang, J.-C.; Khalil, A. M.; Hull, N. P.; Dickson, R. M. FRET-Enabled Optical Modulation for High Sensitivity Fluorescence Imaging. J. Am. Chem. Soc. 2010, 132 (18), 6318-6323.

(17) Díaz, S. a; Giordano, L.; Jovin, T. M.; Jares-Erijman, E. a. Modulation of a Photoswitchable Dual-Color Quantum Dot Containing a Photochromic FRET Acceptor and an Internal Standard. Nano Lett. 2012, 12 (7), 3537-3544.

(18) Coronado, E. A.; Encina, E. R.; Stefani, F. D. Optical Properties of Metallic Nanoparticles: Manipulating Light, Heat and Forces at the Nanoscale. Nanoscale 2011, 3 (10), 4042-4059.

(19) Govorov, A. O. A. O.; Richardson, H. H. H. Generating Heat with Metal Nanoparticles. Nano Today 2007, 2 (1), 30-38.

(20) Encina, E. R.; Coronado, E. A. Plasmon Coupling in Silver Nanosphere Pairs. J. Phys. Chem. C 2010, 114 (9), 3918-3923.

(21) Urban, A. S.; Fedoruk, M.; Horton, M. R.; Rädler, J. O.; Stefani, F. D.; Feldmann, J. Controlled Nanometric Phase Transitions of Phospholipid Membranes by Plasmonic Heating of Single Gold Nanoparticles. Nano Lett. 2009, 9 (8), 2903-2908.

(22) Pustovalov, V. K.; Smetannikov, A. S. Analytical and Computer Modelling of Thermal Processes of Laser Interaction with a Single Nanoparticle. RSC Adv. 2014, 4 (99), 55760-55772.

(23) Baffou, G.; Polleux, J.; Rigneault, H.; Monneret, S. SuperHeating and Micro-Bubble Generation around Plasmonic Nanoparticles under Cw Illumination. J. Phys. Chem. C 2014, 118 (9), 4890-4898.

(24) Baffou, G.; Quidant, R. Thermo-Plasmonics: Using Metallic Nanostructures as Nano-Sources of Heat. Laser Photon. Rev. 2013, 7 (2), 171-187.

(25) Skirtach, A. G.; Dejugnat, C.; Braun, D.; Susha, A. S.; Rogach, A. L.; Parak, W. J.; Möhwald, H.; Sukhorukov, G. B. The Role of Metal Nanoparticles in Remote Release of Encapsulated Materials. Nano Lett. 2005, 5 (7), 1371-1377.

(26) Stehr, J.; Hrelescu, C.; Sperling, R. a; Raschke, G.; Wunderlich, M.; Nichtl, A.; Heindl, D.; Kürzinger, K.; Parak, W. J.; Klar, T. A.; et al. Gold NanoStoves for Microsecond DNA Melting Analysis. Nano Lett. 2008, 8 (2), 619-623.

(27) Adleman, J. R.; Boyd, D. a; Goodwin, D. G.; Psaltis, D. Heterogenous Catalysis Mediated by Plasmon Heating. Nano Lett. 2009, 9 (12), 4417-4423.

(28) Boyer, D.; Tamarat, P.; Maali, A.; Lounis, B.; Orrit, M. Photothermal Imaging of Nanometer-Sized Metal Particles Among Scatterers. Science 2002, 297 (5584), 1160-1163.

(29) Gobin, A. M.; Lee, M. H.; Halas, N. J.; James, W. D.; Drezek, R. a; West, J. L. Near-Infrared Resonant Nanoshells for Combined Optical Imaging and Photothermal Cancer Therapy. Nano Lett. 2007, 7 (7), 1929-1934. 
(30) Valeur, B. Molecular Fluorescence Principles and Applications; Wiley-VCH, 2002.

(31) Jenness, J. R. Effect of Temperature upon the Fluorescence of Some Organics Solutions. Phys. Rev. 1929, 34, 1275-1285.

(32) Tawde, N. R.; Ramanathan, N. Fluorescence of Dyestuff Solutions: Viscosity and Temperature Effects. Proc. Phys. Soc., London, Sect. B 1952, 65 (1), 33-40.

(33) Jenness, J. Effect of Temperature upon the Fluorescence of Some Organic Solutions. Phys. Rev. 1929, 34, 1275-1285.

(34) Bur, A.; Vangel, M.; Roth, S. Fluorescence Based Temperature Measurements and Applications to Real Time Polymer Processing. Polym. Eng. Sci. 2001, 41 (8), 1380.

(35) Ross, D.; Gaitan, M.; Locascio, L. E. Temperature Measurement in Microfluidic Systems Using a Temperature-Dependent Fluorescent Dye. Anal. Chem. 2001, 73 (17), 4117-4123.

(36) Deprédurand, V.; Miron, P.; Labergue, a; Wolff, M.; Castanet, G.; Lemoine, F. A Temperature-Sensitive Tracer Suitable for TwoColour Laser-Induced Fluorescence Thermometry Applied to Evaporating Fuel Droplets. Meas. Sci. Technol. 2008, 19 (10), 105403.

(37) Estrada-Pérez, C. E.; Hassan, Y. a; Tan, S. Experimental Characterization of Temperature Sensitive Dyes for Laser Induced Fluorescence Thermometry. Rev. Sci. Instrum. 2011, 82 (7), 74901.

(38) Baffou, G.; Kreuzer, M. P.; Kulzer, F.; Quidant, R. Temperature Mapping near Plasmonic Nanostructures Using Fluorescence Polarization Anisotropy. Opt. Express 2009, 17 (5), 3291-3298.

(39) Park, K.; Biswas, S.; Kanel, S.; Nepal, D.; Vaia, R. A. Engineering the Optical Properties of Gold Nanorods: Independent Tuning of Surface Plasmon Energy, Extinction Coe Ffi Cient, and Scattering Cross Section. J. Phys. Chem. C 2014, 118 (11), 5918-5926.

(40) Natrajan, V. K.; Christensen, K. T. Two-Color Laser-Induced Fluorescent Thermometry for Microfluidic Systems. Meas. Sci. Technol. 2009, 20 (1), 15401.

(41) Mackey, M. a; Ali, M. R. K.; Austin, L. a; Near, R. D.; El-Sayed, M. a. The Most Effective Gold Nanorod Size for Plasmonic Photothermal Therapy: Theory and in Vitro Experiments. J. Phys. Chem. B 2014, 118 (5), 1319-1326.

(42) Neumann, O.; Urban, A. S.; Day, J.; Lal, S.; Nordlander, P.; Halas, N. J. Solar Vapor Generation Enabled by Nanoparticles. ACS Nano 2013, 7 (1), 42-49.

(43) Mayilo, S.; Kloster, M. A.; Wunderlich, M.; Lutich, A.; Klar, T. A.; Nichtl, A.; Kürzinger, K.; Stefani, F. D.; Feldmann, J. Long-Range Fluorescence Quenching by Gold Nanoparticles in a Sandwich Immunoassay for Cardiac Troponin T. Nano Lett. 2009, 9 (12), $4558-4563$. 